\title{
RNA packaging motor: From structure to quantum mechanical modelling and sequential-stochastic mechanism
}

\author{
Jelena Telenius $^{\mathrm{a} 1}$, Anders E. Wallin ${ }^{\mathrm{b}}$, Michal Straka ${ }^{\mathrm{c} 2}$, Hongbo Zhang ${ }^{\mathrm{a}}$, Erika J. Mancini ${ }^{\mathrm{d}}$ \\ and Roman Tuma ${ }^{\mathrm{a}, \mathrm{e}} *$ \\ ${ }^{a}$ Institute of Biotechnology, University of Helsinki, Helsinki, Finland; ${ }^{b}$ Department of Physics, University \\ of Helsinki, Helsinki, Finland; ${ }^{c}$ Department of Chemistry, University of Helsinki, Helsinki, Finland; \\ ${ }^{d}$ The Wellcome Trust Centre for Human Genetics, University of Oxford, Oxford, UK; ${ }^{e}$ Astbury Centre for \\ Structural Molecular Biology, University of Leeds, Leeds, UK
}

(Received 30 March 2008; final version received 28 April 2008)

\begin{abstract}
The bacteriophages of the Cystoviridae family package their single stranded RNA genomic precursors into empty capsid (procapsids) using a hexameric packaging ATPase motor (P4). This molecular motor shares sequence and structural similarity with RecA-like hexameric helicases. A concerted structural, mutational and kinetic analysis helped to define the mechanical reaction coordinate, i.e. the conformational changes associated with RNA translocation. The results also allowed us to propose a possible scheme of coupling between ATP hydrolysis and translocation which requires the cooperative action of three consecutive subunits. Here, we first test this model by preparing hexamers with defined proportions of wild type and mutant subunits and measuring their activity. Then, we develop a stochastic kinetic model which accounts for the catalytic cooperativity of the P4 hexamer. Finally, we use the available structural information to construct a quantum-chemical model of the chemical reaction coordinate and obtain a detailed description of the electron density changes during ATP hydrolysis. The model explains the results of the mutational analyses and yields new insights into the role of several conserved residues within the ATP binding pocket. These hypotheses will guide future experimental work.
\end{abstract}

Keywords: virus; assembly; molecular motor; density functional; mutagenesis; ATPase

\section{Introduction}

During the life cycle of every virus, its genome needs to be condensed and enclosed within the confines of its capsid. Many RNA viruses utilize a mechanism in which the coat protein subunits and the genomic RNA co-assemble into the viral particle. A classical example is the tobacco mosaic virus in which protein subunits co-assemble with the ssRNA genome into a helical rod $[12,13]$. However, dsDNA viruses typically package their genomes into preformed empty capsid precursors. A well studied example is that of bacteriophage $\phi 29$ in which the packaging is performed by a large molecular motor composed of capsid-bound dodecameric connector, several small RNA subunits and transiently associated ATPase subunits $[28,59,62]$.

dsRNA bacteriophages of the Cystoviridae family (phages $\phi 6-\phi 14)$ utilize the latter mechanism and package ssRNA precursors into preformed procapsids (also called polymerase complexes, Figure 1(A)) [26]. Cystoviruses possess three genomic segments (small, medium

\footnotetext{
*Corresponding author. Email: r.tuma@leeds.ac.uk
} 


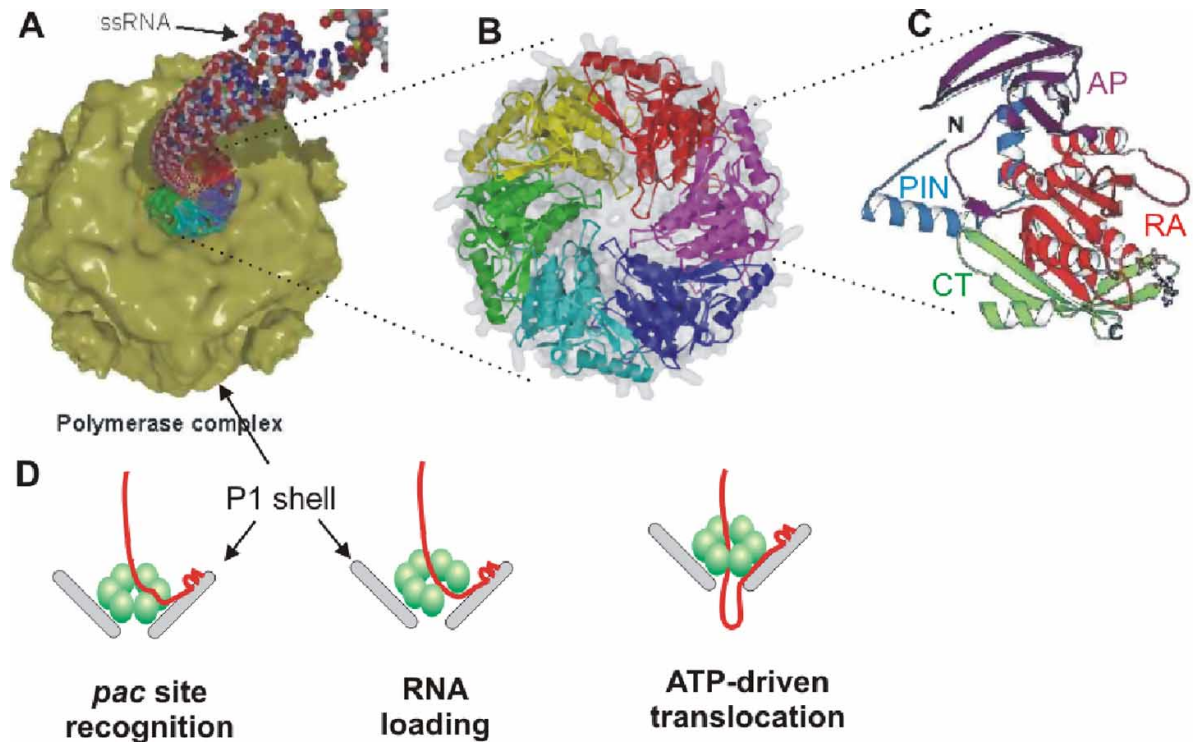

Figure 1. (A) Packaging of ssRNA (spacefill model) via P4 hexamer (ribbon diagram at one vertex, subunits depicted in different colours) into the icosahedral procapsid (khaki surface representation of EM derived model, E. Mancini, unpublished). (B) P4 hexamer structure (C) Subunit structure, RecA-like catalytic core is shown in red (RA), N-terminal safety pin in cyan (PIN), apical domain in magenta (AP) and the C-terminal platform in green (CT). The safety pin stabilizes interacts with a neighbouring subunit and stabilizes the hexamer. (D) A scheme of the three step packaging mechanism for a ssRNA precursor. Only one five-fold vertex and the associated P4 hexamer are shown. In principle, all vertices on the capsid may package RNA, although some evidence for the use of a single vertex has been presented [54].

and large) which are packaged sequentially by the viral packaging motor P4 [52,55] (Figure 1(B)). P4 hexamers [33] reside at each of the five-fold vertices of the procapsid (Figure 1(A)) [27]. After successful packaging, the three ssRNAs are converted into dsRNA inside the viral procapsid by a virus-associated RNA-dependent RNA polymerase (protein P2) [11,25]. For each segment, the packaging process may be divided into three steps (Figure 1(D)): (i) recognition of the RNA pac site (a packaging signal) by a specific binding site on the surface of the viral capsid (i.e. major structural protein P1) [23,50], (ii) loading of the ssRNA into the packaging motor (most likely by a ring-opening mechanism) [43], (iii) unidirectional, $5^{\prime}-3^{\prime}$ translocation of the captured ssRNA into the procapsid at the expense of ATP hydrolysis [24,38]. This mechanism is somewhat similar to those of dsDNA viruses, albeit the packaging machinery of the latter is more complex. While the architecture of cystoviruses is related to that of viruses of the Reoviridae family, packaging in the latter may differ considerably and involves several non-structural proteins [65]. For example, rotavirus protein NSP2, which is involved in packaging and exhibits helicase activity, is an octameric ATPase belonging to the HIT family and is not related to $\mathrm{P} 4$ [29].

The dome-shaped P4 hexamer has an overall structure and subunit fold similar to those of hexameric helicases (Figure 1(B)-(C)) [46,48]. The subunit structure has three domains: $\mathrm{N}$-terminal apical dome, middle RecA-like ATPase core and C-terminal platform. Interestingly, the same basic fold was also found for a packaging ATPase domain from dsDNA bacteriophage $\mathrm{T} 4$ [64]. Thus, based on structure alone one may be tempted to speculate that all these motor proteins may share certain mechanistic features. In recent years, the P4 protein has become one 

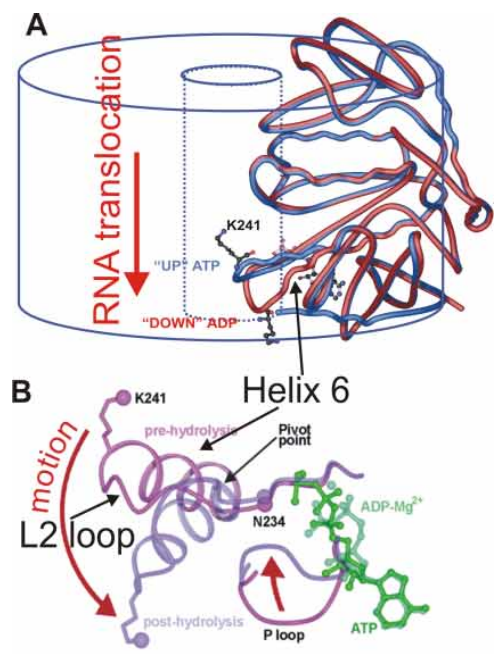

C
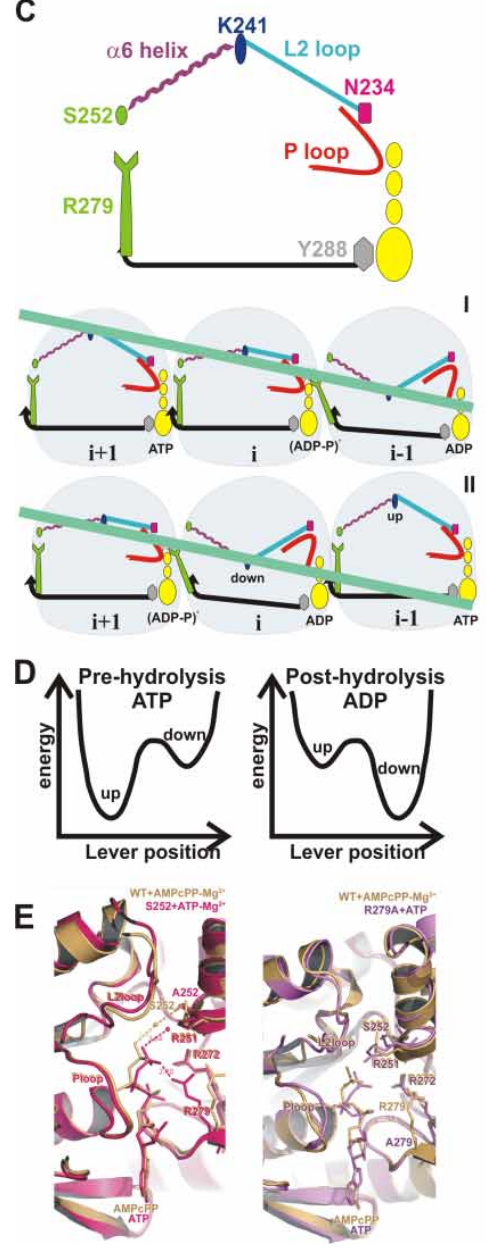

Figure 2. (A-B) Loop L2 movement in the central channel (C) Mechanism of sequential hydrolysis of ATP (yellow) and RNA (light green) translocation for three subunits viewed from within the central 
of the best characterized hexameric helicases and, given the wealth of structural and biophysical information available, it constitutes a good model system for studying the mechano-chemistry of nucleic acid translocation. In this contribution, we first review the available structural and biochemical data together with the proposed model of the mechano-chemical coupling. Then, we present recent experimental and theoretical results and discuss their implications for the model.

P4s from bacteriophages $\phi 6,8,12$ and 13 have been characterized biochemically, crystallized $[45,47]$ and high resolution structures were obtained for some $(\phi 6,12$ and 13) (Figure 1(B)-(C)) $[46,49]$. These proteins can be expressed and purified in hexameric form and possess RNAstimulated ATPase activity [36,38]. Isolated $\phi 8$ and $\phi 13 \mathrm{P} 4$ hexamers also exhibit helicase (duplex unwinding) activity in vitro [38]. The structurally conserved Rec-A like core encompasses five sequence motifs (H1, H1a, H2, H3 and H4) that are common to all superfamily 4 (SF4) helicases $[39,46,48,60]$ (Figure $1(\mathrm{C})$ ). The structural homology of P4 proteins extends to SF3 (human papilloma virus HPV E1, Simian virus 40 SV40 Large T antigen and LTag) and SF5 (Rho terminator) helicases, and to other proteins of the $\mathrm{AAA}^{+}$family [37].

A molecular motor converts chemical energy into mechanical work which is manifested by directional motion. How such conversion is achieved by molecular motors is a matter of considerable interest and debate. In essence, the question is how the progress of the chemical reaction (e.g. ATP binding or hydrolysis in the active site of the ATPase) drives or is coupled to the motion along the mechanical coordinate (e.g. RNA translocation through the hexamer). Two possible scenarios can be envisioned: (i) progress of the chemical reaction leads to small structural changes and charge redistributions (on $\sim 0.1 \mathrm{~nm}$ scale) in the active site that are directly amplified into larger, nanometre scale and movements, (ii) mechanical motion is stochastic and triggers the chemical reaction which in turn biases the movement in one direction. The former scheme is called a power stroke [66] while the latter constitutes a Brownian motor mechanism, of which the Brownian ratchet is a special case [7]. As we argue below, the proposed mechanism for $\mathrm{P} 4$ exhibits features of both the power stroke and the Brownian ratchet.

In order to understand the mechanism of a molecular motor the chemical and mechanical reaction coordinates must be defined and the rules governing coordinated progress and energy exchange between the two coordinates (i.e. mechano-chemical coupling) must be delineated. The first steps towards these goals have been accomplished for $\phi 12 \mathrm{P} 4$. X-ray crystallography revealed that movement of a molecular lever was associated with subtle changes in the nucleotide binding pocket during ATP hydrolysis [46] (Figure 2(A)). The lever consists of two connected elements, an $\alpha$-helix 6 and a loop named L2. The end of the helix inserts into the ATP binding site of a neighbouring subunit, while the loop L2 protrudes into the central channel of the hexamer and returns back to the active site on the same subunit. The L2 loop contains at its centre a lysine (Lys241 in $\phi 12$ P4, Figure 2) which is conserved among most cystoviral P4s [39]. We have recently shown by site-directed mutagenesis that this positively charged side chain interacts with RNA [37]. The position of the L2 loop- $\alpha$-helix 6 lever inside the central channel depends on the occupancy of the ATP binding site. In the presence of AMPcPP, an ATP analogue the lever is in an 'up' position while in the ADP-bound state it is in a 'down' position (Figure 2(A)). The position of the lever ('up' or 'down') correlates with the conformation of the P-loop (H1 motif also known as Walker A), which is seen either retracted from or inserted in the active site (Figure 2(B)). Finally, it was observed that in the 'down' position the side chain

channel. Two consecutive cycles are shown. Top panel contains the key for colour-coding of different conserved residues and the lever elements, helix 6 and L2 loop. (D) Hypothetical energy landscapes for lever motion. (E) Comparison of active site structure with bound ATP (mutant) or AMPcPP (wt, yellow), S252A left and R279A right. Adapted from Ref. [37]. Available in colour online. 
of Arg279 (structurally equivalent to 'arginine finger' in GAPs and F1-ATPase) is inserted into the neighbouring active site, while it is retracted in the 'up position' (Figure 2(E)). In the apo hexamer (i.e. in the absence of bound nucleotide tri- and di-phosphates), the lever can assume both 'up' and 'down' positions suggesting that the nucleotide occupancy controls this movement. The lever movement was also shown to be essential for ATP hydrolysis [37]. Thus, the lever motion could be considered a good approximation for the mechanical reaction coordinate and is tightly coupled to ATP hydrolysis.

By a combination of site-directed mutagenesis and detailed structural analysis we have recently found that the lever motion is directed by the concerted interaction of two sensor residues, Asn234 and Ser252, with the $\gamma$-phosphate $(\gamma \mathrm{P})$ [37]. These sensors belong to helicase motifs H3 (Asn234) and H4 (Ser252), respectively, and are positioned at each end of the lever. They interact with ATP in two neighbouring ATP binding sites. Structural counterparts of these two residues in other helicases have been found to play an important role in the coupling between ATP hydrolysis and DNA binding [14,51,63,68].

The crystal structure of the S252A mutant in complex with ATP and $\mathrm{Mg}^{2+}$ revealed a transient state on the chemical reaction coordinate in which the arginine finger Arg279 is inserted into the catalytic pocket and interacts with the $\gamma \mathrm{P}$ of the bound ATP (Figure 2(E)). Comparing this structure with that of R279A:ATP complex (Figure 2(E)) demonstrated that the arginine finger is not needed for the precise positioning of the $\gamma \mathrm{P}$ inside the binding pocket and likely plays a role later during catalysis, i.e. in formation of the transition state (TS). The S252A structure also revealed that the hydroxyl group of Ser252 is essential for coordinating the catalytic water molecule as in this structure the catalytic water moves away from its usual position next to the $\gamma \mathrm{P}$ (Figure 2(E), left). This observation could also explain why the S252A mutation renders P4 ATPase completely inactive [37].

Biochemical analysis revealed that the ATPase activity and cooperativity in $\mathrm{P} 4$ are increased in the presence of RNA. The cooperativity is catalytic rather than being due to cooperative binding of ATP and is mediated by sequential coordination of hydrolysis between three consecutive subunits (Figure 2(C)) [44]. All structural and biochemical results lead us to put forward a model in which L2 loops of three consecutive subunits bind to the RNA backbone in a staircase configuration similar to that recently revealed for the hexameric helicase E1 of HPV [19]. The three subunits cooperate in sequential hydrolysis (Figure 2(C)) [37]. At the beginning of the cycle (panel I), two subunits (i, i +1 ) are in ATP 'pre-hydrolysis or up' state while the third $(i-1)$ is in the ADP 'product or down' state resulting from the previous hydrolysis cycle. The L2 loop of ( $\mathrm{i}-1)$ is bound to RNA in the 'down' position. In the 'down' position, the arginine finger Arg279 of the (i - 1) is inserted into the (i) active site and triggers or stimulates hydrolysis [37]. The arginine finger insertion constitutes the structural basis for sequential hydrolysis. After hydrolysis at subunit $\mathrm{i}$, the $\mathrm{P}_{\mathrm{i}}$ (inorganic phosphate, $\mathrm{HPO}_{4}^{2-}$ ) is released from the active site and the absence of $\gamma \mathrm{P} / \mathrm{P}_{\mathrm{i}}$ further stabilizes the 'down' position of the preceding (i - 1) lever through the loss of Ser252 side chain interaction with the $\gamma \mathrm{P}$. In this way, the lever motion is being rectified by hydrolysis in the neighbouring active site, i.e. this part of the mechanism is akin to the Brownian ratchet. $P_{i}$ release from active site of (i) also changes the energy landscape for the lever at subunit i due to the loss of interaction between Asn234 side chain and the $\gamma \mathrm{P}$ (Figure 2(B)-(D)). Hence the lever moves to the new, energy favourable 'down' position and propels the bound RNA down the central channel (panel II). This, in turn, may be considered a power stroke albeit the down motion remains fairly stochastic in nature. At the end of the cycle, the (i -1$)$ subunit detaches from RNA, ADP is released and exchanged for ATP to enter another round.

The central idea in this model, the cooperation of three consecutive subunits, has been established and supported through kinetic measurements. While it is in general agreement with the 
structural and mutation analyses, a direct verification is still lacking. One way to probe this model is to generate hexamers harbouring increasing number of mutant (inactive) subunits and measure their specific activity. The current model predicts that the activity would be negligible after reaching the level of three inactive subunits per hexamer. Here, we describe the preparation and activity of such mixed $\phi 12 \mathrm{P} 4$ hexamers harbouring subunits with a mutated arginine finger (R279A).

Modelling the overall kinetics of hexameric helicases is complicated by their high cooperativity and by the number of possible states. Purely kinetic models have now been designed and solved for two hexameric helicases $[1,41]$ however their correspondence with the available structural information is hard to fathom. A structure based approach, i.e. molecular dynamics (MD), offers, in principle, a complete description of the motion [20] but the computational costs for a hexameric system become prohibitive when simulating events on a time scale relevant to enzyme kinetics (i.e. ms). Simplified models based on Brownian dynamics may be used in lieu of full MD description when the trajectory of the mechanical reaction coordinate can be extracted or extrapolated from the structural data $[10,66]$. Here, we use a version of this approach, the discrete event stochastic algorithm [21,22], to simulate the kinetic cooperativity of P4.

Another drawback of MD and related techniques is that they cannot adequately describe the breaking or the forming of covalent bonds, i.e. the phosphodiester bond in ATP [67]. As the latter involves electron density redistributions, it must be described at the quantum mechanical (QM) level. Although QM methods are severely restricted in terms of the size of the system (number of atoms) they can handle, recent developments of density functional theory (DFT) brought within reach the computation of ATPase reaction mechanisms [15,16]. Here, we demonstrate that by judicious use of structural information it is possible to select all important residues in the vicinity of the triphosphate and to obtain a self-consistent model of the active site. This model is then used to map the course of phosphodiester hydrolysis, i.e. the chemical reaction coordinate including the structure and energy of the TS. The roles of the conserved residues in the reaction are examined at the QM level and compared with the results of sitedirected mutagenesis.

The results presented here can be viewed as examples of our current efforts and do not necessarily provide a conclusive picture. Furthermore, greater integration of the theoretical approaches with experimental results is required before we can take full advantage of the models and employ them to test and propose hypotheses.

\section{Experimental}

We prepared hexamers with varying number of mutant subunits by in vitro re-assembly. This approach required several steps: (1) wild type (wt) and mutant hexamer dissociation into subunits, (2) re-assembly and (3) separation of species with a defined number of wt subunits.

In order to aid step 3 , a hexa-histidine tag was added to the $\mathrm{C}$-terminus of the wt protein $\mathrm{P} 4$ from bacteriophage $\phi 12$. The wt gene was PCR-amplified from pPG-27 [47] (pET-32a) with primers containing Nde1 site upstream and Hind3 site downstream and sub-cloned into the Nde1-Hind3 sites of plasmid pET-22b which has T7 promoter and 6 X His Tag 15 base-pairs downstream of the Hind 3 site. The stop codon TGA of $\mathrm{P} 4$ was removed and following primers were used: forward GCCTAA-CATATG-ATCCATCTGTACGACGCA (Nde1); reverse TAATAA-AAGCTTGTTGGAGGTGAGACGACG (Hind3). The resulting plasmid pHB1 was sequenced and introduced into Escherichia coli expression strain BL21 (DE3) [70]. The C-terminally tagged protein (P4His) was expressed at $17^{\circ} \mathrm{C}$ in a similar fashion to wt [47]. P4His was purified following a procedure previously described for his-tagged $\phi 8 \mathrm{P} 4$ protein [6]. The molecular weight of purified protein and presence of his-tag was verified by mass spectrometry. 
Hexamer dissociation was accomplished by diluting P4 protein $(2 \mathrm{mg} / \mathrm{ml}$ final concentration) into a high salt buffer in the absence of divalent cations and nucleotide phosphates (TNE buffer: $20 \mathrm{mM}$ Tris- $\mathrm{HCl}, \mathrm{pH} 8.0,0.5 \mathrm{M} \mathrm{NaCl}, 5 \mathrm{mM}$ EDTA) [34]. The high ionic strength was important for stabilization of the subunits throughout the slow dissociation $\left(48 \mathrm{~h}\right.$ at $\left.25^{\circ} \mathrm{C}\right)$ [70]. Re-association was done by mixing the wt P4His with an untagged arginine finger mutant R279A in the desired proportion. The association products were further enriched in the amount of the desired species by affinity chromatography on Ni-NTA agarose column (HisTrap, GE Healthcare, Freiburg, Germany). Structural integrity of re-assembled hexamers was checked by size-exclusion chromatography coupled with dynamic light scattering [35]. The proportion of wt subunits was estimated from staining of the R279A and wt P4his bands on SDS-PAGE gel using densitometry (Figure 3(A), note that the addition of 6xHis caused P4His to migrate significantly slower). Activity of the purified, mixed hexamers was measured by the EnzChek phosphate release assay as previously described [42].

\section{Theory}

\section{Kinetic model of catalytic cooperativity}

A simplified kinetic model contained six equivalent ATP binding sites flanked by their corresponding levers ( $\alpha 6$ helix-L2 loop) (Figure 4(A) left). ATP and ADP binding and dissociation rate constants were unaffected by the state of neighbouring subunits and lever position (i.e. purely kinetic cooperativity). Since inorganic phosphate $\left(\mathrm{P}_{\mathrm{i}}\right)$ does not seem to inhibit the rate of hydrolysis, we assumed that $P_{i}$ release is fast [44]. Hence we only considered three states for each active site (Figure 4(A) right): empty (E), ATP-bound enzyme substrate (ES), ADP-bound enzyme product (EP). The only reliable set of nucleotide binding and dissociation rate constants was measured for the fluorescently labelled $2^{\prime}$-MANT- $3^{\prime}$-dATP and MANT-ADP analogues. These analogues exhibit higher affinity and thus the apparent Michaelis constant of $2^{\prime}$-MANT-3'-dATP is lower than that of ATP $[42,44]$. Under the steady-state conditions, ADP does not accumulate and hence we set the ADP re-binding constant to zero $(\mathrm{k} 6=0)$.

Very little is known about the magnitudes of intrinsic rate constants for the chemical reaction ( $\mathrm{k} 3$ and $\mathrm{k} 4$ ). From results obtained for related hexameric helicases, this step is likely to be faster than other steps, including the phosphate release step [32]. In the absence of RNA, the overall turnover of P4 is relatively slow $\left(0.8 \mathrm{~s}^{-1}\right.$ for ATP and $0.07 \mathrm{~s}^{-1}$ for $2^{\prime}$-MANT- $3^{\prime}$-dATP [44]) and hence there must be a rate-limiting step prior to the chemical reaction. Given the results of structural and mutational analysis, we think it is fair to assume that the insertion of the arginine finger is the rate-limiting step and a prerequisite for hydrolysis. Hence the intrinsic rate constant, when this step has been fulfilled (i.e. when the lever position in the preceding subunit reached the intermediate state, see below), was set $\mathrm{k} 3=5000 \mathrm{~s}^{-1}$. This means that we consider this step to be almost instantaneous compared with other steps when the prerequisite slower mechanical motion has been achieved. Conversely, in the absence of Arg279 insertion we set k3 $=0$. For simplicity, we set $\mathrm{k} 4=0$, since under the steady-state conditions $\mathrm{P}_{\mathrm{i}}$ release was assumed to be fast, making the reaction virtually irreversible.

In an ideal case, the mechanical part of the cycle, i.e. the lever position, should be modelled as stochastic motion on energy landscapes similar to those shown in Figure 2(D). The exact shape and parameters of these landscapes are not known at the present. Hence, we simplified the trajectory by three points (states) for which there is a structural evidence (Figure 4(A) right): the initial 'up' position, as seen in the P4:AMPcPP: $\mathrm{Mg}^{2+}$ crystal structure (up), an intermediate position (in) which is concomitant with the insertion of the arginine finger and triggers hydrolysis in the neighbour (e.g. configuration similar to that in the S252A:ATP: $\mathrm{Mg}^{2+}$ structure 

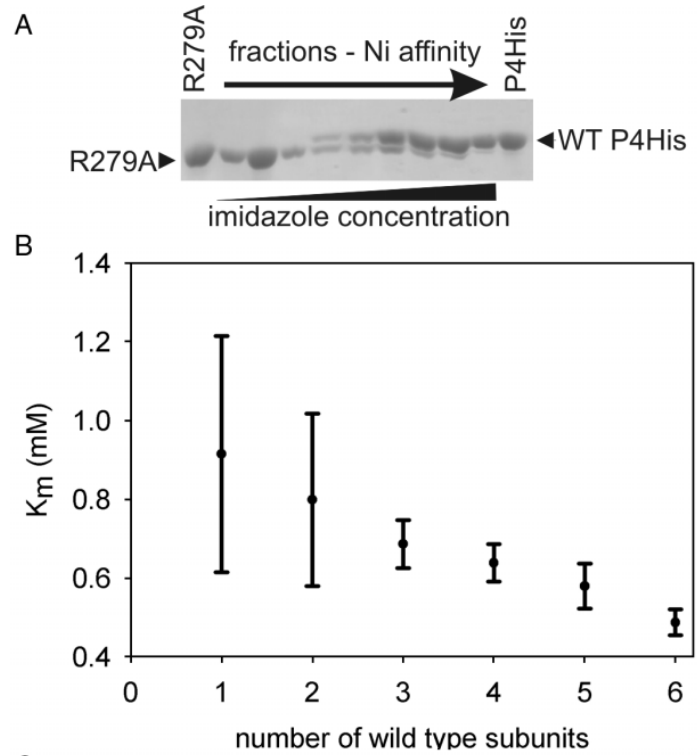

C

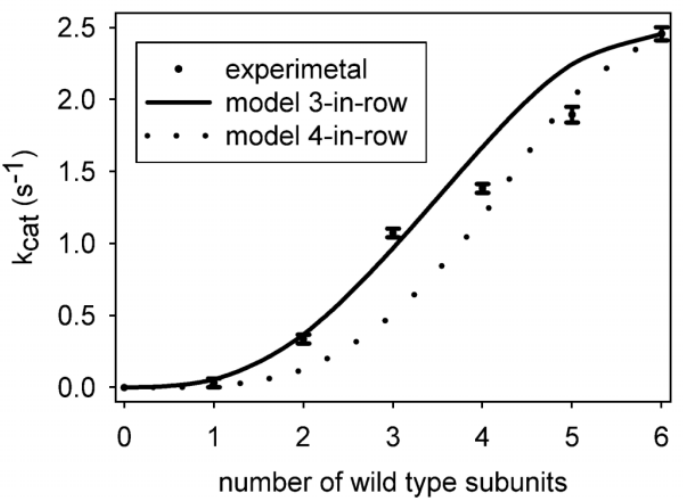

D

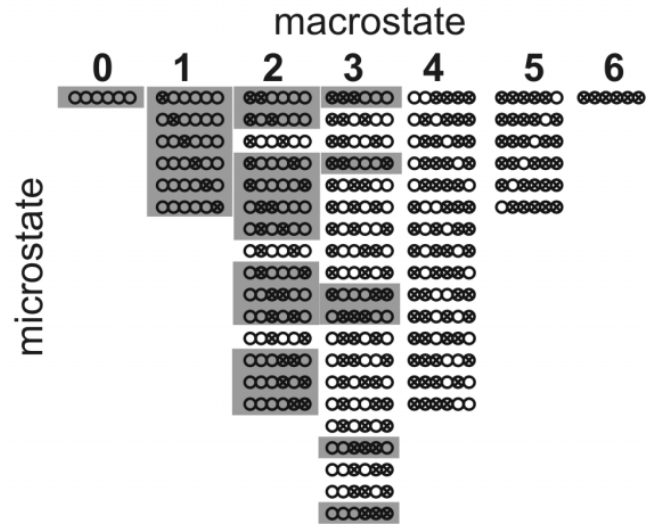

Figure 3. Incorporation of increasing number of arginine finger mutant (R279A) subunits into hexamers. (A) Separation of hexamers by Ni-affinity chromatography. Note the slower migration of wt P4His due to the addition of the 6xHis and the intervening linker residues. (B) Dependence of the Michaelis constant on the number of wt subunits (from initial mixing ratio). (C) Dependence of the turnover number on the 

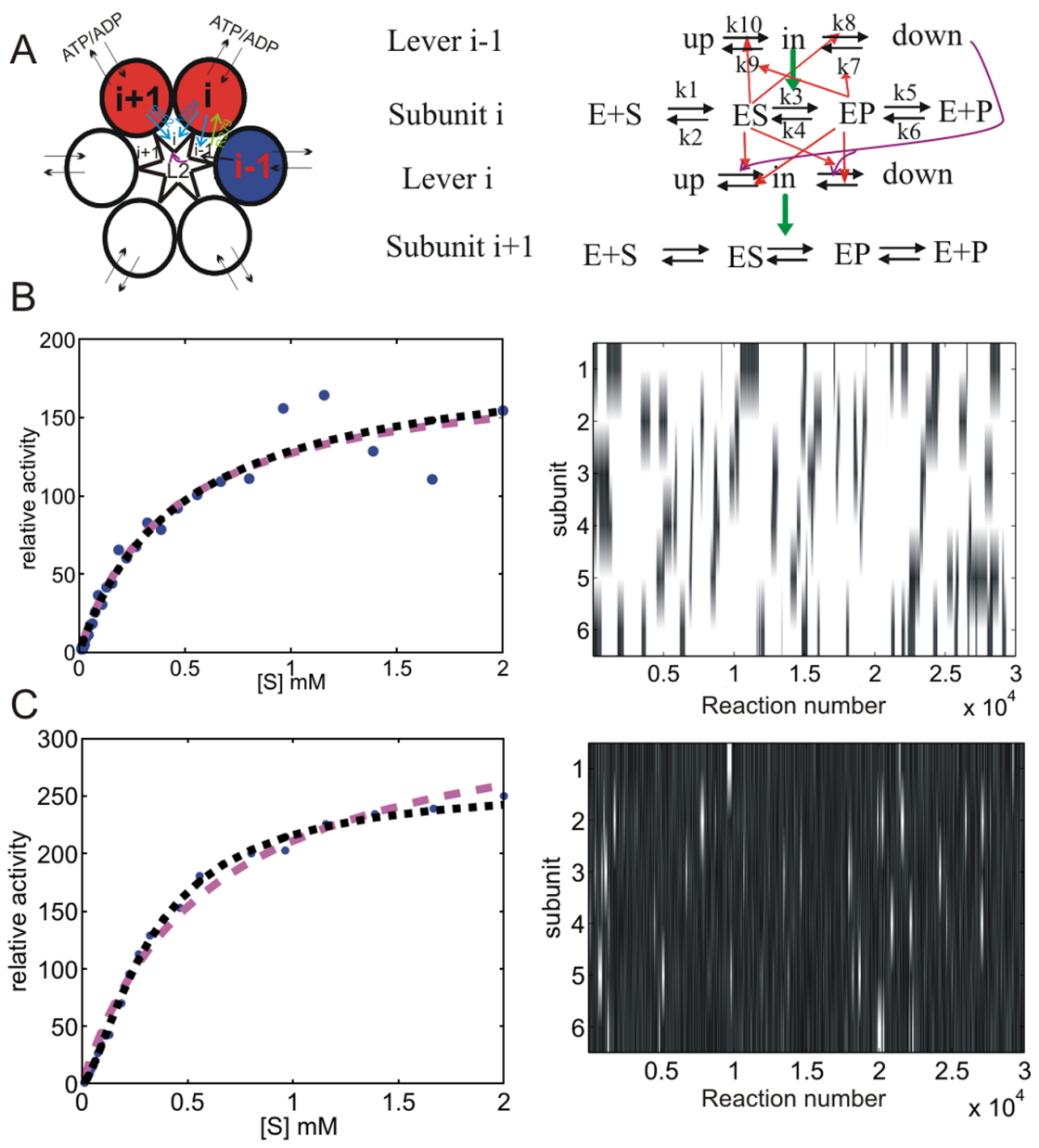

Figure 4. (A) Schematics of the ATP binding sites interconnecting mechanical levers (L2) and the key residues that control the lever position depending on $\gamma \mathrm{P}$ presence (left). In the right panel, the overall scheme of reaction is given for the levers (up, in and down states) and ATP binding sites belonging to three consecutive subunits (S, ATP; ES, substrate complex; EP, product complex; P, product). The stimulating effect on hydrolysis in the next neighbour is shown as green arrows while slowing down the lever motion is depicted as red arrows. RNA binding to the neighbouring subunits causes synchronization of motion of their levers. This is modelled as an increase of the lever motion rate constant depending on the neighbouring lever position (magenta arrow). (B) Simulated relative activity as a function of MANT-ATP concentration (left) and occupancy of states during the simulation (right) in the absence of RNA. Apparent $K_{\mathrm{m}}=0.41$ and $k_{\max }=163$. (C) As in panel B but in the presence of RNA, $K_{\mathrm{m}}=0.27$ and $k_{\max }=249 n=1.6$. In each left panel, the dashed magenta curve represents a simple hyperbolic fit (Michaelis-Menten non-cooperative kinetics) while the black curve is fit to Hill equation $V=k_{\max } *[\mathrm{ATP}]^{n} /\left([\mathrm{ATP}]^{n}+K_{\mathrm{m}}^{\mathrm{n}}\right)$. In the right panels, the lever states are coded as follows: up, white; down, black; in, grey. Available in colour online.

number of wt subunits. The expected turnover numbers for the three-in-row and four-in-row models are shown as solid and dashed curves, respectively. Error bars (SD) were estimated from several independent determinations. (D) Enumeration of different microstates for the six macrostates (from Ref. [44]). Active microstates for the three-in-row model are highlighted. 

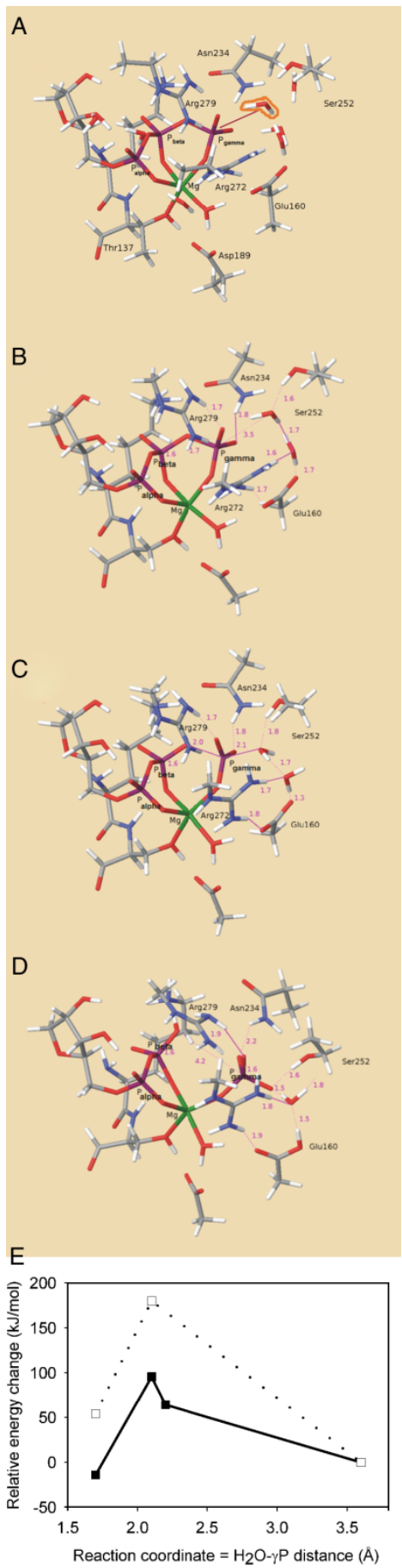

Figure 5. Structural snapshots along the reaction coordinate obtained by QM modeling. QM optimized model of ES state with all atoms shown (A) and with omitted methyl groups and unimportant water molecules (B). Structure close to the TS state (C) and in product state EP with $\mathrm{P}_{\mathrm{i}}$ still bound (D). Energy along the reaction coordinate for the wt (solid line and filled symbols) and R279A mutant (dotted line, empty symbols). The ES and EP states were fully optimized without any constraints while the bond length 
in Figure 2(E) but closer to the TS structure, see next section on QM modelling for details), and the 'down' position, as seen in the P4:ADP: $\mathrm{Mg}^{2+}$ structure (down). The inter-conversions between these states were modelled as regular chemical reactions. The inter-conversion rates for the lever at subunit $i$ were modulated by the nucleotide binding state at this (through interaction of Asn234 with the $\gamma \mathrm{P})$ and the following subunit $(\mathrm{i}+1$, via Ser252 interaction with the $\gamma \mathrm{P})$. The presence of these interactions slowed the 'down' motion ten-fold while their absence slowed the 'up' motion (Figure 4(A) right). In the absence of nucleotides, we assumed fast interconversion rates since reasonable results were obtained with $\mathrm{k} 7-10 \sim 50-100 \mathrm{~s}^{-1}$. Obviously, lever motion is the least determined feature of the model and needs further improvements, e.g. by extracting energy landscapes from molecular mechanics simulations or estimating the lever mobility constants from experiment such as NMR spectroscopy.

RNA binds to Lys241 at the tip of the moving lever [37] and was modelled as a link between two consecutive levers (Figure 2(D)). Motion of one lever facilitates, via the RNA link, the neighbouring lever to adopt the same position, i.e. accelerates the lever motion in one direction ten-fold. Once again this is a somewhat arbitrary number since there is no information on the kinetics of RNA binding and correlation of lever positions. RNA unbinding from one subunit was followed by its binding to a neighbour (Figure 2(D)).

The overall scheme of coupled reactions is summarized in Figure 4(A). Obviously, such a coupled system would be difficult to solve exactly and hence we employed the stochastic discrete step simulation, as originally devised by Gillespie [21]. This scheme employs variable time steps and is implemented in a Matlab shell which allows for easy modifications and improvements. Further acceleration of simulation was accomplished by implementing the core algorithm in $\mathrm{C}$ as a subroutine. Steady-state kinetics rates were obtained from linear fits of product concentrations versus time for 1000 simulation steps at each of the 30 different ATP concentrations, without and with RNA present. Using the stochastic simulation approach, we can in the future extend the scheme to include Brownian motion of the lever in a measured or computed potential.

\section{QM modelling of the chemical reaction}

\section{Model of the enzyme-substrate complex}

The model was constructed by combining the available crystallographic structures of the P4 active site $[37,46]$. The missing hydrogen atoms, which are not visible in the X-ray structure at $2 \AA$ resolution, were subsequently added by applying the correct stereochemistry. The resulting system was truncated to a computationally tractable model by a careful selection of the important residues while including as a large part of the reaction centre as possible. For comparison, much smaller regions are usually treated quantum mechanically in the QM/MM calculations $[15,17]$.

In more detail, the model of the active site was constructed by combining two crystal structures of bacteriophage $\phi 12$ motor protein P4. Structure 1W49 (PDB id. 1W49) (Protein Data Bank: [9]) presents P4 protein in complex with an ATP-analogue, AMPcPP and $\mathrm{Mg}^{2+}$ [46]. The 2VHQ (PDB id. 2VHQ) structure is a mutant P4 in which Ser252 was replaced with alanine, and complexed with ATP and $\mathrm{Mg}^{2+}$ [37]. This combination was devised to obtain ATP in an approximation of its native, hydrolysable form, since in the wt complex the bound AMPcPP

along the reaction coordinate was held constant during the TS state optimization. Molden, version 4.5 beta [57], gOpenMol, version 3.00 [8,40] and Maestro (Copyright 1999-2007 Schrödinger, LLC) packages were employed for vizualization of the results. Available in colour online. 
is in an inactive configuration (see comparison in Figure 2(E)). Figure 5(A) illustrates the merged structure in which the ATP-enzyme complex is ready for hydrolysis. The arginine finger (Arg279), which was also taken from 2VHQ, is in the hydrolysis stimulating, inserted position, i.e. close to the $\gamma \mathrm{P}$. Ser 252 and the catalytically important water molecule were taken from 1W49 structure since they were missing from the mutant [37]. The P-loop amino acids (Lys136 and Thr137) were poorly resolved in the 2VHQ structure, and hence taken from the 1W49 wt structure. All other amino acids were taken from 2VHQ structure to get the model as close as possible to the situation just before ATP hydrolysis (Figure 5(A)).

The most conserved water molecules in the active site were found with the help of UCSF Chimera modelling environment [53]. The active sites of the three subunits in the asymmetric unit were aligned with each other in both crystal structures and water molecules present in at least two subunits were included in the model. In total, eight water molecules were found within the active centre (Figure 5(A)). Hydrogen atoms were added using an automatic procedure implemented in Maestro (v.8.0) and further checked manually in order to assure proper ionization forms of the amino acids, assuming the local $\mathrm{pH}$ in the active site to be between 7 and 8 , as in the experiments or in vivo conditions.

To obtain a computationally feasible system, several amino acids were truncated at a location unlikely to directly influence the reaction: The backbone regions and hydrocarbon (aliphatic) portions of all amino acids, except the P-loop (Lys136 and Thr137), were replaced with methyl groups. The resulting truncated system (Figure 5(B)) was then used in all calculations.

\section{DFT and computational details}

In the DFT, the electron cloud is described by one electron density function, which includes all electrons of the system, rather than one wave function for each individual electron [31]. Thus, a $3 \mathrm{~N}$-dimensional N-electron problem is reduced to a three dimensional problem and makes DFT calculations fast and scaleable. The price to pay for this advantage is that the exact form of the density functional is not known and various approximations are necessary.

The quantum-chemical calculations were done with Turbomole software package [3], versions 5.8. The RI-J (resolution of the identity) and MARI-J (multipole accelerated resolution of the identity) DFT schemes were employed to accelerate the calculations [58]. Split-valence polarization (def-SVP) basis sets were used for all atoms [56], except magnesium, for which the more appropriate triple-zeta valence polarization set was used [69]. Corresponding auxiliary basis sets for RI approximation were used [18]. Most calculations were done with PerdewBurke-Ernzerhof (PBE) functional and confirmed with the hybrid B3LYP (Becke 3-parameter exchange functional with Lee-Yang-Parr correlation) functional. Default code parameters were used except for the finer numerical integration accuracy in DFT (gridsize m4). Relaxed convergence criteria were used for obtaining the complete reaction coordinate with reasonable numerical accuracy.

\section{Results and discussion}

\section{A minimum of three subunits in a row cooperate in hydrolysis}

Mutation of the arginine finger Arg279 to alanine (R279A) causes severe reduction of the P4 ATPase activity ( $>1000$-fold) [37]. We have incorporated increasing numbers of R279A subunits into the hexamer by mixing it with wt his-tagged subunits (P4His). Separation of the hexamers according to the increasing number of P4His subunits is demonstrated in Figure 3(A). Nickel affinity purification and gel staining also demonstrated that the proportion of the wt 
subunits in the peak fraction follows the initial mixing ratio (not shown). Hence, we subsequently prepared hexamers by simply mixing different proportions of dissociated subunits followed by size-exclusion chromatography to remove unassembled subunits or residual aggregates. The ratio of wt and mutant subunits was verified by SDS-PAGE as described above.

The steady-state kinetic parameters (apparent Michaelis constant, $K_{\mathrm{m}}$, and the turnover number $k_{\text {cat }}$ in the presence of $1 \mathrm{mM}$ poly $(\mathrm{rC})$ ) for hexamers at different mixing ratios are shown in Figure 3(B)-C. With the increasing number of mutated, inactive subunits both $k_{\text {cat }}$ and $K_{\mathrm{m}}$ were affected. Although the R279A mutation does not affect the ATP affinity [37] $K_{\mathrm{m}}$ increased roughly linearly with the number of inactive subunits incorporated (Figure 3(B)). This is clear manifestation of catalytic (kinetic) cooperativity.

As expected from the proposed cooperativity model (i.e. three consecutive subunits cooperate, three-in-row model), $k_{\text {cat }}$ decreased to almost background noise levels when the number of incorporated mutated subunits was more than three. The observed residual activity is due to binomial distribution of the number of inactive subunits in mixed hexamers (hexameric macrostates $0-6$ in Figure 3(D)) at each mixing ratio (probabilities related to the initial mixing ratio $p=[\mathrm{wt}] /([\mathrm{wt}]+[\mathrm{R} 275 \mathrm{~A}]), q=1-p)$. Assuming that the mutation does not affect hexamer assembly (the structure of R279A mutant is essentially wt [37]), each macrostate is composed of equally populated microstates which are the configurations of inactive subunits within the hexamer (Figure 3(D)). For a given mechanism, certain microstates are active while others are not [44]. For example, the active configurations for the three-in-row mechanism are highlighted in Figure 3(D). By counting the number of active configurations for each macrostate and weighting them by the binomial distribution of macrostates, we obtained the expected activity as a function of number of wt subunits (see [44] for details). Two models, corresponding to the three-in-row and the four-in-row model, respectively, are compared with experimental data in Figure 3(C). At low numbers of mutant subunits, the experimental points follow the fourin-row model while at higher numbers they are closer to the three-in-row model. This suggests that during fast hydrolysis and processive translocation (i.e. at ATP saturation levels) up to four subunits in row may cooperate. The hexamer can however still work with a minimum of three subunits in a row, as suggested previously [37,44]. More sophisticated models are needed to fully account for the experimental data.

\section{Kinetic simulation of stochastic-sequential cooperativity}

One of the hallmarks of $\mathrm{P} 4$ cooperativity is the apparently high Michaelis constant $\left(K_{\mathrm{m}}>K_{\mathrm{d}}\right)$ in the absence of RNA which becomes comparable to the equilibrium dissociation constant $\left(K_{\mathrm{d}}\right)$ when RNA is bound and translocated. In addition, cooperativity is clearly discernible in the presence of RNA and is accompanied by a modest increase of $k_{\text {cat }}$ (three-fold for ATP and about ten-fold for MANT-ATP). Note that RNA has no effect on ATP or ADP binding kinetics and equilibrium [44]. Standard kinetic models do not account for this behaviour. Here, we present a stochastic-sequential model (Figure 4(A)) which predicts with reasonable accuracy the expected decrease in $K_{\mathrm{m}}$ and increase in $k_{\text {cat }}$ and cooperativity (compare Figure 4(B),(C)). However, the activity enhancement was less than expected for MANT-ATP (note MANT-ATP was used in lieu of ATP for which no binding rate constants are known at the present time). The discrepancy is not surprising given that some of the kinetic constants in the model could only be estimated.

Comparison of lever state distributions throughout the entire course of the simulation provided additional insight (Figure 4(B),(C), right panels). There was a dramatic difference in the pattern of lever motion and therefore hydrolysis rate in absence or presence of RNA. Without RNA only isolated, stochastic 'islands' of sequential hydrolysis are apparent and the subunits spend most of the time waiting for the right constellation of binding sites and levers to re-start 
sequential hydrolysis. RNA binding introduces coupling of lever motion between neighbouring subunits and as a consequence hydrolysis becomes sequential and processive. The proposed simulation scheme constitutes a plausible starting model. Since variations in the estimated rate constants and their couplings yield different results we need to obtain further constraints from experiment.

\section{QM model of the chemical reaction coordinate}

\section{Structure of ATP-bound state}

While the 'pre-hydrolysis' model structure after optimization at the QM level correlates well with the equivalent atomic structure based model, the packing of amino acids in the active site in the former is slightly denser than that in the latter. This may be due to missing protein environment around the active centre. In both the atomic structure based model and the QM optimized model structure, Ser252 coordinates a water molecule which then attacks the $\gamma \mathrm{P}$ during the reaction. In the QM optimized model (Figure 5(A)), Lys136 side chain in the P loop was closer to the $\beta$-phosphate ( $\beta P$ ) than in the atomic structure based model. Similarly, Glu160, which coordinates another important water molecule (the so called assisting water), moved closer to the $\gamma \mathrm{P}$ following the rearrangement of water molecules in the vicinity of $\gamma \mathrm{P}$ during optimization. Also, Arg279 and Arg272 moved closer to the $\gamma \mathrm{P}$ and rotated around their side chain axes. The conformation of ATP remained almost the same as in the 2VHQ structure. All three phosphates form coordination bonds with the magnesium ion, which is also coordinated by one water molecule and the oxygen of Thr137 hydroxyl group.

\section{Reaction path}

A common mechanism of ATP hydrolysis is a nucleophilic attack of a water molecule on the $\gamma \mathrm{P}[2,15]$. Hence, we initiated the reaction by moving 'in silico' the catalytic water molecule (highlighted in Figure 5(A)) towards the $\gamma \mathrm{P}$ and optimizing the system at each $\mathrm{H}_{2} \mathrm{O}-\mathrm{P} \gamma$ distance. The energy profile along this reaction coordinate exhibited a typical maximum due to formation of a high-energy TS followed by a sharp drop to the lower energy products (Figure 5(E)). The TS energy barrier was $90 \mathrm{~kJ} / \mathrm{mol}$, somewhat higher than the experimental value $(63 \mathrm{~kJ} / \mathrm{mol})$ obtained for the related $\phi 6[30]$ and other ATPases. The model yielded also structural information about the TS and the product state with $\mathrm{P}_{\mathrm{i}}$ still present within the binding pocket. Both of these states are short-lived and thus hard to study experimentally. Starting from the reactant state in Figure 5(B), the reaction proceeded via a TS in which the $\gamma \mathrm{P}$ was in a typical penta-coordinated configuration (Figure 5(C)) [2]. Following the TS, the $\gamma \mathrm{P}$ separated abruptly from the $\beta \mathrm{P}$ while still being coordinated to $\mathrm{Mg}^{2+}$ (Figure 5(D)). After the $\gamma \mathrm{P}$ separation, Lys136 lowered the high negative charge of ADP by donating a proton to the $\beta \mathrm{P}$ (Table 1).

In addition, the computational method offers insights which are not easily obtainable from experimental methods, such as unravelling the detailed path to the TS and the proton relay involved in generation of the nucleophilic agent, the hydroxyl anion, from the attacking water molecule. The nucleophilicity of the water molecule was increased by an assisting water molecule that accepts a proton from the attacking water and becomes a positively charged hydronium ion. The hydronium ion in turn sheds the extra proton to the carboxylate of Glu160 which becomes a neutral carboxylic acid (Figure 5(D) and Table 1). The formation of the hydroxyl anion was concomitant with its approach to the $\gamma \mathrm{P}$. The assisting water molecule remained hydrogen-bonded to the oxygen on the $\mathrm{P}_{\mathrm{i}}$ which originated from the attacking water (Figure 5(D)). 
Table 1. Calculated natural charges along the reaction path.

\begin{tabular}{lrrr}
\hline Residues & \multicolumn{1}{c}{ ES } & TS & EP \\
\hline Ser252 & -0.06 & -0.01 & -0.01 \\
Glu160 & -0.71 & -0.69 & -0.04 \\
Arg272 & 0.73 & 0.80 & 0.78 \\
Arg279 & 0.79 & 0.79 & 0.78 \\
Thr137 & -0.14 & -0.18 & -0.17 \\
Lys136 & 0.76 & 0.83 & 0.12 \\
Asn234 & -0.03 & -0.03 & -0.06 \\
Asp189 & -0.68 & -0.71 & -0.70 \\
Mg & 1.73 & 1.76 & 1.73 \\
ATP phosphates & -3.53 & -3.73 & -3.63 \\
$\alpha P+\beta P$ & -1.99 & -2.70 & -2.08 \\
$\gamma P$ & -1.54 & -1.03 & -1.55 \\
Ribose & 0.28 & 0.29 & 0.29 \\
\hline
\end{tabular}

ES, enzyme substrate complex; TS, transition state; EP, enzyme product complex.

\section{Role of arginine fingers in catalysis}

An arginine finger is a residue which, at various points along the catalytic cycle, inserts into the active site of many GTPases and ATPases and generally accelerates the hydrolytic reaction. Arginine fingers were first identified in small GTPases (G-proteins) where they are provided in trans by activator proteins (GAPs) [4]. For G-proteins, the catalytic role of arginine fingers has been firmly established [4,5]. Since arginine fingers always coordinate the $\gamma \mathrm{P}$ of NTP, it has also been proposed that in hexameric rings they act as $\gamma \mathrm{P}$ sensors which relay the information from one NTP binding site to the adjacent subunit and therefore promote sequential conformational changes $[19,61]$.

P4 has two arginine residues in the active site, Arg272 and Arg279 (Figure 5(A)). Although Arg272 is essential for ATPase activity [37], it does not fulfil the role of a finger since it is not being inserted into the active site during the cycle [46]. It probably does not act as a sensor either. Indeed, the computational model confirmed that it has a catalytic role instead of sensory one. Arg272 is important for the formation of the attacking hydroxyl ion and coordinates the assisting water molecule (Figure 5(C)).

Arg279 side chain is inserted into the active site during the course of the reaction and it interacts with the $\gamma \mathrm{P}$. ATP binding kinetic is not affected by the R279A mutation [37] indicating a catalytic rather than sensory role. Indeed, the computational model demonstrated that the Arg279 side chain offers a positive charge to the negatively charged exiting $\mathrm{P}_{\mathrm{i}}$. Further insight was gained by computing the reaction path for the R279A mutant. Compared to wt, the energy barrier almost doubled (Figure 5(E)) and the product state was less stable, i.e. making the intrinsic hydrolysis an unfavourable reaction. These results indicate that Arg279 may play a dual role, the first is in the TS formation and the second in stabilizing the product state before $\mathrm{P}_{\mathrm{i}}$ release. While the stabilization of $\mathrm{P}_{\mathrm{i}}$ may slow down its release, which incidentally is the ratelimiting step for many helicases, it may allow the chemical reaction to proceed within the centre making it slightly energetically favourable. It remains to be seen whether the $P_{i}$ release is assisted by further motion of the arginine finger towards the $\alpha \mathrm{P}$ and $\beta \mathrm{P}$ with which it interacts in the crystal structure of the product P4:ADP: $\mathrm{Mg}^{2+}$ state.

\section{Conserved serine within helicase motif $\mathrm{H} 4$ has dual role in the mechanism}

The hydroxyl group of Ser252, which is positioned at the end of the moving helix 6, is coordinating the attacking water molecule at all stages of the hydrolysis reaction (Figure 5). 
The hydrolysis reaction and movement of helix 6 are thus connected via Ser252. In the simulation Ser252 hydroxyl group followed the attacking water deeper into the active site and helped to align the catalytic water for nucleophilic attack (Figure 5(C)). After reaching the TS, the serine withdrew from the active site (Figure 5(D)) but remained coordinated to the bound $\mathrm{P}_{\mathrm{i}}$. Ser252 is likely to retreat to its resting position during or after the release of $\mathrm{P}_{\mathrm{i}}$. As the backbone of Ser252 follows the side chain motion this could provide a mechanism by which the energy from $\mathrm{P}_{\mathrm{i}}$ release is harnessed to effect mechanical motion, e.g. a small movement and turning of Ser 252 could cause the whole helix 6 to pivot like a see-saw. This mechanism may also work in the opposite direction, triggering hydrolysis by a coordinated insertion of Ser252 hydroxyl and Arg279 finger upon helix 6 motion. Taken together, these results suggest both sensory and catalytic role for Ser252 in P4 mechanism. It is tempting to propose that this dual role is conserved among all the helicases with structurally related serine or histidine residues [17].

In conclusion, we have devised a concerted experimental and theoretical approach to investigate the complex mechanisms behind the cooperativity and mechano-chemical coupling of hexameric molecular motors. Whilst some of the theoretical tools are still rather rudimentary, e.g. the stochastic kinetic model, others are more mature and provide insights inaccessible to experiment. Theoretical tools, hand-in-hand with new experimental approaches will play essential role in understanding these fascinating molecular machines.

\section{Acknowledgements}

This work was supported by Academy of Finland (award 118462 to R.T.). Anders Wallin is supported by Nanoscience Graduate School, Finland. M.S. was supported by a Marie Curie Intra-European Fellowships within the European Community 6th Framework Program. Magnus Ehrnrooth Foundation is gratefully acknowledged for travel support of M.S. Jelena Telenius is supported by National Graduate School in Materials Physics and by the Helsinki University of Technology. Erika Mancini is supported by the Royal Society. Computational resources were provided by CSC Scientific Computing Ltd., Espoo, Finland.

\section{Notes}

1. Present address: Laboratory of Physics, Helsinki University of Technology, Helsinki, Finland.

2. Present address: Institute of Organic Chemistry and Biochemistry, Academy of Sciences of the Czech Republic, Prague, Czech Republic.

\section{References}

[1] J.L. Adelman, Y.J. Jeong, J.C. Liao, G. Patel, D.E. Kim, G. Oster, and S.S. Patel, Mechanochemistry of transcription termination factor Rho, Mol. Cell 22 (2006), pp. 611-621.

[2] S.J. Admiraal and D. Herschlag, Mapping the transition state for ATP hydrolysis: Implications for enzymatic catalysis, Chem. Biol. 2 (1995), pp. 729-739.

[3] R. Ahlrichs, M. Bar, M. Haser, H. Horn, and C. Kolmel, Electronic structure calculations on workstation computers: The program system turbomole, Chem. Phys. Lett. 162 (1989), pp. 165-169.

[4] M.R. Ahmadian, P. Stege, K. Scheffzek, and A. Wittinghofer, Confirmation of the arginine-finger hypothesis for the GAP-stimulated GTP-hydrolysis reaction of Ras, Nat. Struct. Biol. 4 (1997), pp. 686-689.

[5] C. Allin, M.R. Ahmadian, A. Wittinghofer, and K. Gerwert, Monitoring the GAP catalyzed H-Ras GTPase reaction at atomic resolution in real time, Proc. Natl. Acad. Sci. USA 98 (2001), pp. 7754-7759.

[6] Y. Astier, D.E. Kainov, H. Bayley, R. Tuma, and S. Howorka, Stochastic detection of motor proteinRNA complexes by single-channel current recording, Chemphyschem 8 (2007), pp. 2189-2194.

[7] R.D. Astumian, Thermodynamics and kinetics of a Brownian motor, Science 276 (1997), pp. 917-922.

[8] D.L. Bergman, L. Laaksonen, and A. Laaksonen, Visualization of solvation structures in liquid mixtures, J. Mol. Graph Model 15 (1997), pp. 301-306, 328-33. 
[9] H.M. Berman, T.N. Bhat, P.E. Bourne, Z. Feng, G. Gilliland, H. Weissig, and J. Westbrook, The protein data bank and the challenge of structural genomics, Nat. Struct. Biol. 7(Suppl.) (2000), pp. 957-959.

[10] C. Bustamante, D. Keller, and G. Oster, The physics of molecular motors, Acc. Chem. Res. 34 (2001), pp. 412-420.

[11] S.J. Butcher, J.M. Grimes, E.V. Makeyev, D.H. Bamford, and D.I. Stuart, A mechanism for initiating RNA-dependent RNA polymerization, Nature 410 (2001), pp. 235-240.

[12] P.J. Butler, J.T. Finch, and D. Zimmern, Configuration of tobacco mosaic virus, RNA during virus assembly, Nature 265 (1977), pp. 217-219.

[13] P.J. Butler and G.P. Lomonossoff, RNA-protein interactions in the assembly of tobacco mosaic virus, Biophys. J. 32 (1980), pp. 295-312.

[14] D.J. Crampton, S. Mukherjee, and C.C. Richardson, DNA-induced switch from independent to sequential dTTP hydrolysis in the bacteriophage T7 DNA helicase, Mol. Cell 21 (2006), pp. 165-174.

[15] M. Dittrich, S. Hayashi, and K. Schulten, On the mechanism of ATP hydrolysis in F1-ATPase, Biophys. J. 85 (2003), pp. 2253-2266.

[16] — ATP hydrolysis in the betaTP and betaDP catalytic sites of F1-ATPase, Biophys. J. 87 (2004), pp. 2954-2967.

[17] M. Dittrich and K. Schulten, PcrA helicase, a prototype ATP-driven molecular motor, Structure 14 (2006), pp. 1345-1353.

[18] K. Eichkorn, O. Treutler, H. Öhm, M. Häser, and R. Alrichs, Auxiliary basis sets to approximate coulomb potentials, Chem. Phys. Lett. 242 (1995), pp. 652-660.

[19] E.J. Enemark and L. Joshua-Tor, Mechanism of DNA translocation in a replicative hexameric helicase, Nature 442 (2006), pp. 270-275.

[20] Y.Q. Gao, W. Yang, and M. Karplus, A structure-based model for the synthesis and hydrolysis of ATP by F1-ATPase, Cell 123 (2005), pp. 195-205.

[21] D.T. Gillespie, Exact stochastic simulation of coupled chemical-reactions, J. Phys. Chem. 81 (1977), pp. 2340-2361.

[22] $\longrightarrow$ Monte-Carlo simulation of random-walks with residence time-dependent transitionprobability rates, J. Comp. Phys. 28 (1978), pp. 395-407.

[23] P. Gottlieb, X. Qiao, J. Strassman, M. Frilander, and L. Mindich, Identification of the packaging regions within the genomic RNA segments of bacteriophage phi 6, Virology 200 (1994), pp. 42-47.

[24] P. Gottlieb, J. Strassman, A. Frucht, X.Y. Qiao, and L. Mindich, In vitro packaging of the bacteriophage phi 6 ssRNA genomic precursors, Virology 181 (1991), pp. 589-594.

[25] P. Gottlieb, J. Strassman, X.Y. Qiao, A. Frucht, and L. Mindich, In vitro replication, packaging, and transcription of the segmented double-stranded RNA genome of bacteriophage phi 6: Studies with procapsids assembled from plasmid-encoded proteins, J. Bacteriol. 172 (1990), pp. 5774-5782.

[26] J.T. Huiskonen, F. de Haas, D. Bubeck, D.H. Bamford, S.D. Fuller, and S.J. Butcher, Structure of the bacteriophage phi6 nucleocapsid suggests a mechanism for sequential RNA packaging, Structure 14 (2006), pp. 1039-1048.

[27] J.T. Huiskonen, H.T. Jaalinoja, J.A. Briggs, S.D. Fuller, and S.J. Butcher, Structure of a hexameric RNA packaging motor in a viral polymerase complex, J. Struct. Biol. 158 (2007), pp. 156-164.

[28] B. Ibarra, J.M. Valpuesta, and J.L. Carrascosa, Purification and functional characterization of p16, the ATPase of the bacteriophage Phi29 packaging machinery, Nucleic Acids Res. 29 (2001), pp. 4264-4273.

[29] H. Jayaram, Z. Taraporewala, J.T. Patton, and B.V. Prasad, Rotavirus protein involved in genome replication and packaging exhibits a HIT-like fold, Nature 417 (2002), pp. 311-315.

[30] R.H. Jenkins, R. Tuma, J.T. Juuti, D.H. Bamford, and G.J. Thomas, Jr., A novel Raman spectrophotometric method for quantitative measurement of nucleoside triphosphate hydrolysis, Biospectroscopy 5 (1999), pp. 3-8.

[31] F. Jensen, Introduction to Computational Chemistry, John Wiley \& Sons, Chichester, 2007.

[32] Y.J. Jeong, D.E. Kim, and S.S. Patel, Kinetic pathway of dTTP hydrolysis by hexameric T7 helicaseprimase in the absence of DNA, J. Biol. Chem. 277 (2002), pp. 43778-43784.

[33] J.T. Juuti and D.H. Bamford, RNA binding, packaging and polymerase activities of the different incomplete polymerase complex particles of dsRNA bacteriophage phi 6, J. Mol. Biol. 249 (1995), pp. 545-554.

[34] J.T. Juuti, D.H. Bamford, R. Tuma, and G.J. Thomas, Jr., Structure and NTPase activity of the RNA-translocating protein (P4) of bacteriophage phi 6, J. Mol. Biol. 279 (1998), pp. 347-359. 
[35] D.E. Kainov, S.J. Butcher, D.H. Bamford, and R. Tuma, Conserved intermediates on the assembly pathway of double-stranded RNA bacteriophages, J. Mol. Biol. 328 (2003), pp. 791-804.

[36] D.E. Kainov, J. Lisal, D.H. Bamford, and R. Tuma, Packaging motor from double-stranded RNA bacteriophage phil 2 acts as an obligatory passive conduit during transcription, Nucleic Acids Res. 32 (2004), pp. 3515-3521.

[37] D.E. Kainov, E.J. Mancini, J. Telenius, J. Lisal, J.M. Grimes, D.H. Bamford, D.I. Stuart, and R. Tuma, Structural basis of mechano-chemical coupling in a hexameric molecular motor, J. Biol. Chem. 283 (2008), pp. 3607-3617.

[38] D.E. Kainov, M. Pirttimaa, R. Tuma, S.J. Butcher, G.J. Thomas, Jr., D.H. Bamford, and E.V. Makeyev, RNA packaging device of double-stranded RNA bacteriophages, possibly as simple as hexamer of P4 protein, J. Biol. Chem. 278 (2003), pp. 48084-48091.

[39] D.E. Kainov, R. Tuma, and E.J. Mancini, Hexameric molecular motors: P4 packaging ATPase unravels the mechanism, Cell. Mol. Life Sci. 63 (2006), pp. 1095-1105.

[40] L. Laaksonen, A graphics program for the analysis and display of molecular dynamics trajectories, J. Mol. Graph. 10 (1992), pp. 33-34, 24.

[41] J.C. Liao, Y.J. Jeong, D.E. Kim, S.S. Patel, and G. Oster, Mechanochemistry of $t 7$ DNA helicase, J. Mol. Biol. 350 (2005), pp. 452-475.

[42] J. Lisal, D.E. Kainov, D.H. Bamford, G.J. Thomas, Jr., and R. Tuma, Enzymatic mechanism of RNA translocation in double-stranded RNA bacteriophages, J. Biol. Chem. 279 (2004), pp. 1343-1350.

[43] J. Lisal, T. Lam, D.E. Kainov, M.R. Emmett, A.G. Marshall, and R. Tuma, Functional visualization of viral molecular motor by hydrogen-deuterium exchange reveals transient states, Nat. Struct. Mol. Biol. 12 (2005), pp. 460-466.

[44] J. Lisal and R. Tuma, Cooperative mechanism of RNA packaging motor, J. Biol. Chem. 280 (2005), pp. 23157-23164.

[45] E.J. Mancini, J.M. Grimes, R. Malby, G.C. Sutton, D.E. Kainov, J.T. Juuti, E.V. Makeyev, R. Tuma, D.H. Bamford, and D.I. Stuart, Order and disorder in crystals of hexameric NTPases from dsRNA bacteriophages, Acta Crystallogr. D 59 (2003), pp. 2337-2341.

[46] E.J. Mancini, D.E. Kainov, J.M. Grimes, R. Tuma, D.H. Bamford, and D.I. Stuart, Atomic snapshots of an RNA packaging motor reveal conformational changes linking ATP hydrolysis to RNA translocation, Cell 118 (2004), pp. 743-755.

[47] E.J. Mancini, D.E. Kainov, H. Wei, P. Gottlieb, R. Tuma, D.H. Bamford, D.I. Stuart, and J.M. Grimes, Production, crystallization and preliminary $X$-ray crystallographic studies of the bacteriophage phi 12 packaging motor, Acta Crystallogr. D 60 (2004), pp. 588-590.

[48] E.J. Mancini and R. Tuma, Structure and function of P4, a dsRNA virus packaging motor, in Segmented Double-Stranded RNA Viruses. Structure and Molecular Biology, J. T. Patton, ed., Caister Academic Press U.K., Norfolk, 2008.

[49] C. Meier, E.J. Mancini, D.H. Bamford, M.A. Walsh, D.I. Stuart, and J.M. Grimes, Overcoming the false-minima problem in direct methods: Structure determination of the packaging enzyme P4 from bacteriophage phi13, Acta Crystallogr. D 61 (2005), pp. 1238-1244.

[50] L. Mindich, Packaging, replication and recombination of the segmented genome of bacteriophage Phi6 and its relatives, Virus Res. 101 (2004), pp. 83-92.

[51] T. Niedenzu, D. Roleke, G. Bains, E. Scherzinger, and W. Saenger, Crystal structure of the hexameric replicative helicase RepA of plasmid RSF1010, J. Mol. Biol. 306 (2001), pp. 479-487.

[52] A.O. Paatero, L. Mindich, and D.H. Bamford, Mutational analysis of the role of nucleoside triphosphatase P4 in the assembly of the RNA polymerase complex of bacteriophage phi6, J. Virol. 72 (1998), pp. 10058-10065.

[53] E.F. Pettersen, T.D. Goddard, C.C. Huang, G.S. Couch, D.M. Greenblatt, E.C. Meng, and T.E. Ferrin, UCSF Chimera - a visualization system for exploratory research and analysis, J. Comp. Chem. 25 (2004), pp. 1605-1612.

[54] M.J. Pirttimaa, A.O. Paatero, M.J. Frilander, and D.H. Bamford, Nonspecific nucleoside triphosphatase P4 of double-stranded RNA bacteriophage phi6 is required for single-stranded RNA packaging and transcription, J. Virol. 76 (2002), pp. 10122-10127.

[55] X. Qiao, J. Qiao, and L. Mindich, Stoichiometric packaging of the three genomic segments of doublestranded RNA bacteriophage phi6, Proc. Natl Acad. Sci. USA 94 (1997), pp. 4074-4079.

[56] A. Schaefer, H. Horn, and R.J. Ahlrichs, Fully optimized contracted Gaussian basis sets for atoms Li to Kr, J. Chem. Phys. 97 (1992), pp. 2751-2777.

[57] G. Schaftenaar and J.H. Noordik, Molden: A pre- and post-processing program for molecular and electronic structures, J. Comp. Aided Mol. Design 14 (2000), pp. 123-134. 
[58] M. Sierka, A. Hogekamp, and R. Ahlrichs, Fast evaluation of the Coulomb potential for electron densities using multipole accelerated resolution of identity approximation, J. Chem. Phys. 118 (2003), pp. 9136-9148.

[59] A.A. Simpson, Y. Tao, P.G. Leiman, M.O. Badasso, Y. He, P.G. Jardine, N.H. Olson, M.C. Morais, S. Grimes, and D.L. Anderson et al., Structure of the bacteriophage phi29 DNA packaging motor, Nature 408 (2000), pp. 745-750.

[60] M.R. Singleton, M.S. Dillingham, and D.B. Wigley, Structure and mechanism of helicases and nucleic acid translocases, Annu. Rev. Biochem. 76 (2007), pp. 23-50.

[61] M.R. Singleton, M.R. Sawaya, T. Ellenberger, and D.B. Wigley, Crystal structure of 77 gene 4 ring helicase indicates a mechanism for sequential hydrolysis of nucleotides, Cell 101(6) (2000), pp. 589-600.

[62] D.E. Smith, S.J. Tans, S.B. Smith, S. Grimes, D.L. Anderson, and C. Bustamante, The bacteriophage phi29 portal motor can package DNA against a large internal force, Nature 413 (2001), pp. 748-752.

[63] P. Soultanas and D.B. Wigley, Site-directed mutagenesis reveals roles for conserved amino acid residues in the hexameric DNA helicase DnaB from Bacillus stearothermophilus, Nucleic Acids Res. 30 (2002), pp. 4051-4060.

[64] S. Sun, K. Kondabagil, P.M. Gentz, M.G. Rossmann, and V.B. Rao, The structure of the ATPase that powers DNA packaging into bacteriophage T4 procapsids, Mol. Cell 25 (2007), pp. 943-949.

[65] Z.F. Taraporewala and J.T. Patton, Nonstructural proteins involved in genome packaging and replication of rotaviruses and other members of the Reoviridae, Virus Res. 101 (2004), pp. 57-66.

[66] H. Wang and G. Oster, Ratchets, power strokes, and molecular motors, Appl. Phys. A 75 (2002), pp. 315-323.

[67] A. Warshel, Computer Modeling of Chemical Reactions in Enzymes and Solutions, John Wiley \& Sons, New York, 1997.

[68] M.T. Washington, A.H. Rosenberg, K. Griffin, F.W. Studier, and S.S. Patel, Biochemical analysis of mutant $T 7$ primase/helicase proteins defective in DNA binding, nucleotide hydrolysis, and the coupling of hydrolysis with DNA unwinding, J. Biol. Chem. 271 (1996), pp. 26825-26834.

[69] F. Weigend and R. Alrichs, Balanced basis sets of split valence, triple zeta valence and quadruple zeta valence quality for $H$ to Rn: Design and accuracy, Phys. Chem. Chem. Phys. 7 (2005), pp. 3297-3305.

[70] H. Zhang, Contribution of arginine finger to cooperativity in hexameric viral ATPase, MBIOT, Master of Science, University of Helsinki, Helsinki, 2008. 


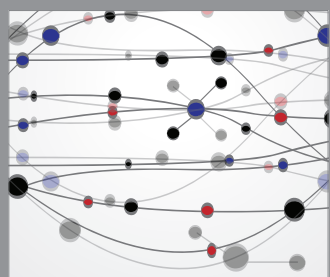

The Scientific World Journal
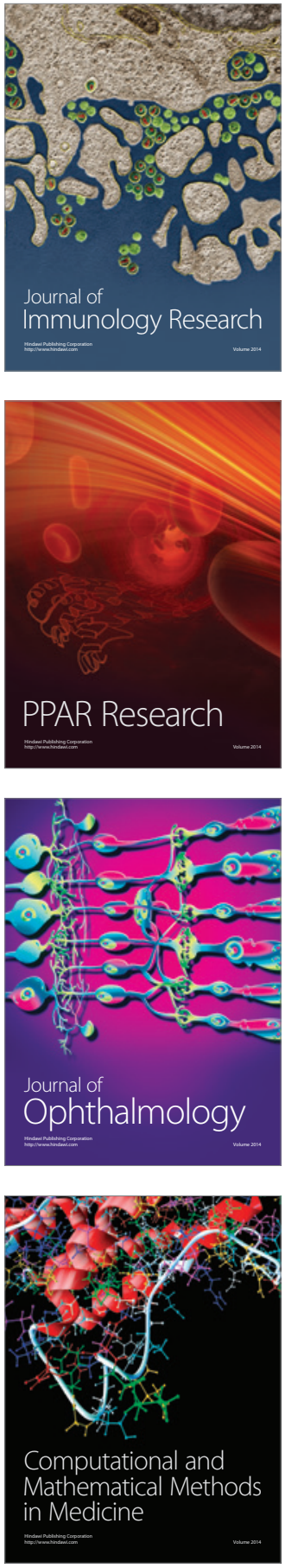

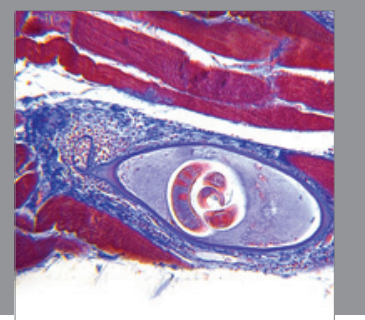

Gastroenterology

Research and Practice
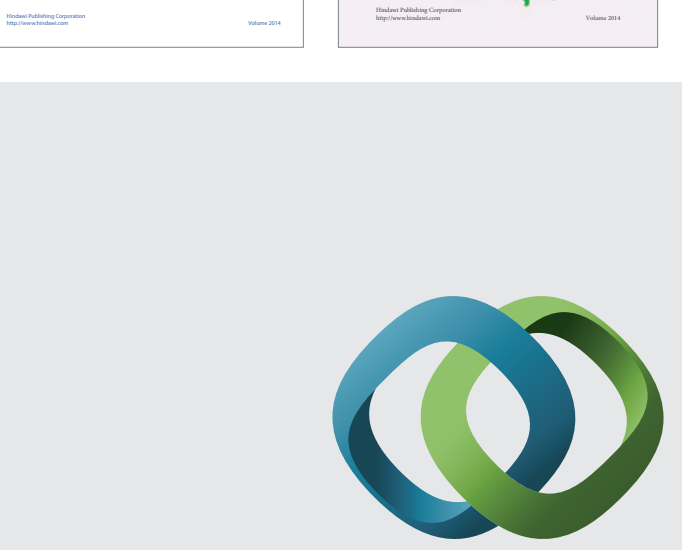

\section{Hindawi}

Submit your manuscripts at

http://www.hindawi.com
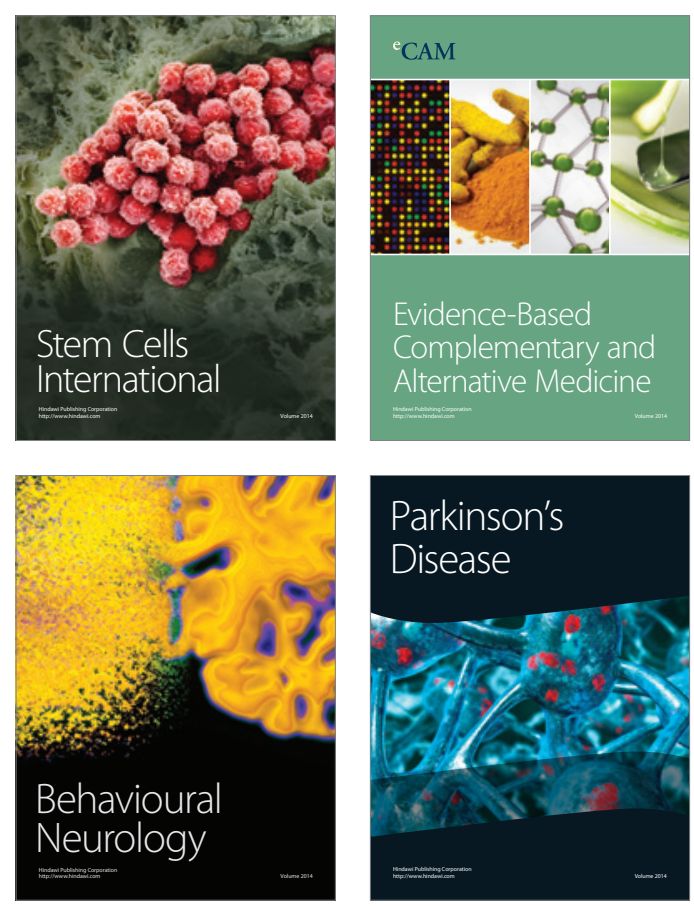

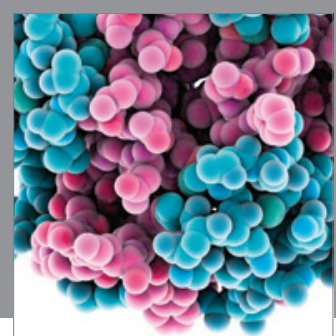

Journal of
Diabetes Research

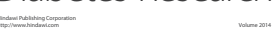

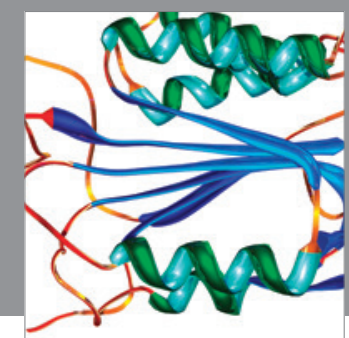

Disease Markers
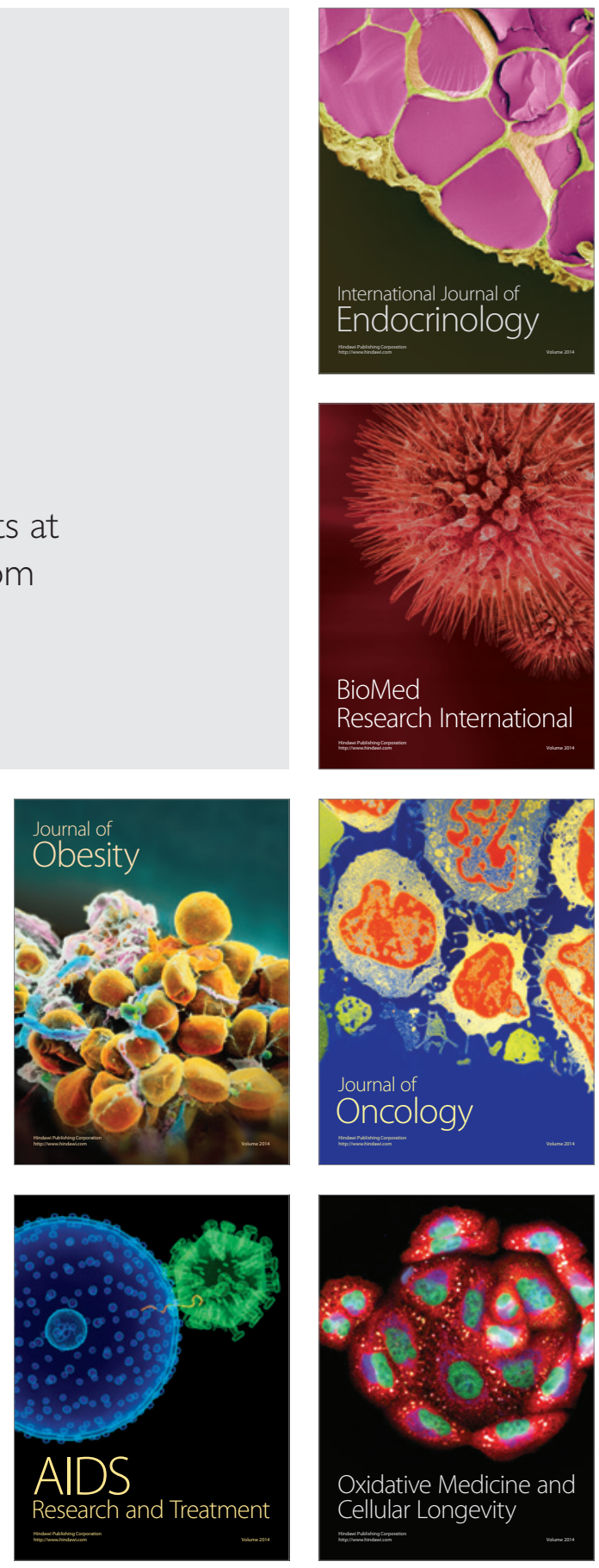